\title{
ERRATUM
}

\section{Erratum for Chirehwa et al., Model-Based Evaluation of Higher Doses of Rifampin Using a Semimechanistic Model Incorporating Autoinduction and Saturation of Hepatic Extraction}

Maxwell T. Chirehwa, ${ }^{\text {a }}$ Roxana Rustomjee, ${ }^{\text {b }}$ Thuli Mthiyane, ${ }^{\text {c }}$ Philip Onyebujoh, ${ }^{\text {d }}$ Peter Smith, ${ }^{\text {a }}$ Helen Mcllleron, ${ }^{\text {a }}$ Paolo Denti ${ }^{\text {a }}$

Division of Clinical Pharmacology, Department of Medicine, University of Cape Town, Cape Town, South Africa'; Strategic Health Innovation Partnerships (SHIP), South African Medical Research Council, Cape Town, South Africab; TB Research Unit: Clinical and Biomedical, South African Medical Research Council, Durban, South Africa ${ }^{c}$; Intercountry Support Team for East and Southern Africa, World Health Organization Regional Office for Africa, Harare, Zimbabwe ${ }^{d}$

Volume 60, no. 1, p. 487-494, 2016. Page 493: Equation A3 should read as follows:

$$
\mathrm{CL}_{\text {int }}=\frac{\mathrm{CL}_{\text {int,max }} \cdot K_{m}}{C_{H}+K_{m}}
$$

